\title{
LA SUSCEPTIBILIDAD DE LAS BROMELIÁCEAS EPÍFITAS AL CAMBIO CLIMÁTICO
}

\author{
Manuel J. Cach-Pérez $Z^{1}$; José Luis Andrade ${ }^{1,2,3}$ y Casandra Reyes-García ${ }^{1}$ \\ 'Unidad de Recursos Naturales, Centro de Investigación Científica de Yucatán, Mérida, Yucatán, México \\ ${ }^{2}$ Unidad de Ciencias del Agua, Centro de Investigación Científica de Yucatán, Cancún, Quintana Roo, México \\ ${ }^{3}$ Autor para la correspondencia: andrade@cicy.mx
}

\begin{abstract}
Resumen: Las bromeliáceas epífitas son uno de los grupos de plantas más amenazados por el cambio climático global dada su alta sensibilidad a la variación ambiental. En esta revisión se abordan las adaptaciones que poseen las bromeliáceas epífitas para hacer frente a las condiciones ambientales adversas. Asimismo, para brindar un panorama general de su vulnerabilidad, se describen, con ejemplos, las respuestas fisiológicas de estas plantas (principalmente de bromeliáceas epífitas en bosques secos de la Península de Yucatán) ante la variación en la disponibilidad de agua, luz y temperatura ambiental, así como cambios en aspectos morfológicos. La información analizada sugiere que las bromeliáceas epífitas podrían cambiar su distribución o desaparecer de algunas regiones como consecuencia del cambio climático global, lo que repercutiría de manera negativa en los ecosistemas en los que estas plantas habitan, al reducir la introducción de nutrimentos, la disponibilidad de hábitats para animales, así como en la biomasa general de los bosques. Por lo tanto, se sugiere que la investigación actual acerca de la variación morfo-fisiológica de las bromeliáceas epífitas, en respuesta a la variación ambiental, es fundamental y urgente para generar información que contribuya a la conservación de estas plantas.
\end{abstract}

Palabras clave: bosques secos, metabolismo ácido de las crasuláceas, variación morfo-fisiológica, Yucatán.

\begin{abstract}
Epiphytic bromeliads are one of the most threatened groups of plants by global climate change due to its high sensitivity to environmental variation. In this review we discuss the adaptations that epiphytic bromeliads have to face adverse environmental conditions. Also, to provide an overview of their vulnerability, we describe with examples, the physiological responses of these plants (mainly epiphytic bromeliads in dry forests of the Yucatan Peninsula) to the variation in water availability, light and environmental temperature, as well as their morphological variation. The data analyzed suggests that epiphytic bromeliads distribution could change or disappear in some regions as a result of global climate change, which could have negative effects on the ecosystems that these plants inhabit, due to reduction in the introduction of nutrients, the availability of habitats for animals as well as in the overall forest biomass. Therefore, we suggest that current research on morpho-physiological variation of epiphytic bromeliads in response to environmental variation is essential and urgent to generate information that contributes to the conservation of these plants.
\end{abstract}

Key words: Crassulacean acid metabolism, dry forests, morpho-physiological variation, Yucatan.

L as epífitas son plantas que crecen sobre otras plantas, como árboles y arbustos, sin tener contacto directo con los haces vasculares de sus hospederos (Lüttge, 1989; Benzing, 1998), y constituyen un componente importante de las comunidades vegetales, principalmente en el trópico. La importancia de las epífitas radica en su gran diversidad, pues se han estimado alrededor de 30,000 especies. De hecho, en los bosques tropicales, la biomasa epífita puede constituir más del $50 \%$ de la biomasa foliar y el $10 \%$ de todas las especies de plantas vasculares, y en algunos bosques puede llegar a representar hasta el $25 \%$ del total de especies de plantas vasculares (Nadkarni, 1984; Kress, 1986; Gentry y Dodson 1987; Benzing, 1989, 2000; Nieder et al., 2001).

Al no contar con raíces en el suelo, las epífitas tienen una extraordinaria dependencia de la atmósfera para la adquisición de los nutrimentos y el agua que necesitan, lo que las hace excepcionalmente sensibles a la humedad del aire (Benzing, 1990), y por tanto, son potencialmente útiles para 
medir la respuesta a los cambios en el clima (Lugo y Scatena, 1992; Helliker y Griffiths, 2007). Asimismo, dada la importancia ecológica de estas plantas, la alta vulnerabilidad de las epífitas a perturbaciones climáticas podría afectar a otros componentes de los bosques donde habitan, (Zotz y Bader, 2009). Después de Orchidaceae, Bromeliaceae es una de las familias con más diversidad de epífitas, y cuenta con al menos 1,800 especies epífitas, lo que representa el $60 \%$ de todas las especies dentro de la familia (Zotz, 2013; Gentry y Dodson, 1987; Kress, 1986).

En las últimas décadas, ha ido creciendo la idea de que la supervivencia y continuidad de la comunidad de epífitas está en riesgo ante el cambio climático global (Nadkarni, 1992). En particular, las bromeliáceas epífitas son uno de los grupos más amenazados, ya que el sistema radical sólo las adhiere al hospedero, y los nutrimentos y el agua necesarios para su crecimiento son absorbidos por las hojas. A diferencia de otros tipos de epífitas cuyas raíces exploran una mayor área y crecen en sitios con acumulación de suelo rico en nutrimentos (Benzing, 1990; Lesica y Antibus, 1990; Andrade y Nobel, 1996, 1997), las bromeliáceas epífitas dependen en mayor medida de lo que les llega de la atmósfera y lo que se acumule entre sus hojas. Por esto, es primordial entender la respuesta de las bromeliáceas epífitas, tanto a nivel individual como poblacional, ante las fluctuaciones ambientales ocasionadas por el cambio climático global y cómo se podrían afectar los ecosistemas en los que se encuentran. Esta revisión se centra en las variaciones fisiológicas, anatómicas y morfológicas que pueden sufrir las bromeliáceas epífitas como respuesta a uno o varios factores de estrés ambiental, como el incremento de la temperatura y la radiación solar, así como la disminución en la disponibilidad de agua.

\section{¿Por qué son tan sensibles las bromeliáceas epífitas a los cambios en el ambiente?}

Dentro de las condiciones microambientales inherentes a las epífitas, la disponibilidad de agua (que es tomada en pulsos durante los eventos de precipitación pluvial, incluso de neblina y rocío), es el factor ambiental más poderoso de su distribución. Este hecho hace que las epífitas puedan responder más rápidamente a cambios en los patrones de precipitación generados por el cambio climático global y provocar desplazamientos geográficos locales o la desaparición de especies en ciertas áreas (Benzing, 1998).

Estudios del efecto ambiental en la fisiología de las bromeliáceas epífitas muestran que éstas responden a la disponibilidad de agua (en forma de lluvia, neblina o rocío) y a la cantidad de radiación solar que reciben, principalmente con relación a su distribución vertical (Graham y Andrade, 2004; Cervantes et al., 2005; Cach-Pérez, 2008; ReyesGarcía et al., 2012). Por ejemplo, la distribución vertical de Tillandsia brachycaulos y T. elongata, especies que coexisten en la selva baja caducifolia de Yucatán, responde a la sensibilidad y tolerancia de cada una a la radiación solar y disponibilidad de agua: $T$. elongata, que es menos tolerante a la falta de agua que $T$. brachycaulos, se localiza en zonas del dosel mucho más expuestas, donde puede captar mayor cantidad de agua (Graham y Andrade, 2004), a pesar de recibir una mayor irradiación y viento. Además, se ha observado una respuesta de ambas especies a las variaciones climáticas a lo largo del año (Andrade, 2003; Graham y Andrade, 2004; Zotz y Asshoff, 2009), lo que también afecta a nivel poblacional, pues se ha observado una reducción en el número de individuos de T. brachycaulos después de un año anormalmente seco en la mencionada selva baja caducifolia (Mondragón et al., 2004).

El efecto de los cambios ambientales será más evidente en los extremos de las distribuciones geográficas de las especies; es decir, en los lugares más secos y con las temperaturas más extremas donde una especie se encuentre, pues estaría ya al límite de su óptimo fisiológico (Lambers et al., 1998). A este respecto, debido al cambio climático, las selvas secas estacionales, manglares y desiertos podrían ser sitios de mayor recambio en las especies.

Nos encontramos en una época donde en promedio, las temperaturas del hemisferio norte durante la segunda mitad del siglo XX fueron superiores a las de cualquier otro periodo de 50 años de los últimos 500 años, y probablemente las más altas a lo largo de los últimos 1,300 años. Diversos modelos de cambio climático coinciden en que la temperatura seguirá aumentando más o menos uniformemente a nivel global (más notoriamente en el hemisferio norte que en el sur) a una tasa de $0.2{ }^{\circ} \mathrm{C}$ por decenio; además, con un grado de confianza alto, las proyecciones indican que, hacia mediados del siglo, la escorrentía fluvial anual y la disponibilidad de agua aumentarán en latitudes altas (y en ciertas áreas lluviosas tropicales), y disminuirán en algunas regiones secas en latitudes medias y en los trópicos (IPCC, 2007).

\section{El metabolismo ácido de las crasuláceas en las brome- liáceas epífitas}

Una de las principales respuestas evolutivas de plantas que crecen en ambientes áridos o con escasez de agua (como el hábitat epífito), es el metabolismo ácido de las crasuláceas (CAM; siglas en inglés), el cual está presente en muchas especies de bromeliáceas epífitas (Martin, 1994; Benzing, 2000; Andrade et al., 2007, 2009). Éste es una ruta fotosintética que permite a las plantas un uso eficiente del agua. En la fotosíntesis CAM, el intercambio de gases se lleva a cabo durante la noche, cuando la demanda evaporativa de la atmósfera es baja, lo que reduce la pérdida de agua por transpiración (Winter y Smith, 1996). Las plantas que cuentan con fotosíntesis CAM no deben su economía en el uso del agua sólo a la fijación temporal nocturna de $\mathrm{CO}_{2}$ (en forma de ácidos orgánicos), sino a que la enzima fos- 
foenol piruvato carboxilasa (PEPc), encargada de la captación primaria de $\mathrm{CO}_{2}$, tiene una mayor afinidad por dicho gas que la enzima ribulosa bifosfato carboxilasa/oxigenasa (RUBISCO), la fijadora de $\mathrm{CO}_{2}$ en el ciclo de Calvin; por lo que las plantas con CAM pueden fijar mayor cantidad de $\mathrm{CO}_{2}$ en relación a la cantidad de agua perdida durante el intercambio de gases.

La fotosíntesis CAM consta de cuatro fases con características fisiológicas distintivas. Durante la primera (que ocurre cuando los estomas se abren en la noche), la PEPc fija temporalmente el $\mathrm{CO}_{2}$ en forma de ácidos orgánicos que se almacenan dentro de las vacuolas de las células. La fase dos sólo ocurre durante un breve periodo de tiempo al amanecer, cuando hay fijación de $\mathrm{CO}_{2}$ por RUBISCO, después de lo cual los estomas se cierran. En este punto hay una transición por competencia de sustrato entre PEPc y RUBISCO, pero durante el periodo de luz únicamente RUBISCO permanece activa. Una vez cerrados los estomas, los ácidos orgánicos almacenados en las vacuolas son descarboxilados en el citosol durante el día y el $\mathrm{CO}_{2}$ liberado ingresa al ciclo de Calvin para producir azúcares (fase tres). Finalmente, durante la fase cuatro, que ocurre al final del periodo de luz cuando se ha consumido todo el ácido acumulado durante la noche anterior, los estomas se abren e inicia la fijación de $\mathrm{CO}_{2}$ tanto por PEPc como por RUBISCO, esta última cesa su actividad con la obscuridad (Osmond, 1987; Benzing, 2000). Si bien no todas las bromeliáceas epífitas son CAM, este tipo fotosintético predomina en las epífitas de zonas de clima seco o con sequía estacional (Smith et al., 1986a; Andrade, 2003; Graham y Andrade, 2004; Andrade et al., 2007). Se ha observado que muchas especies presentan gran plasticidad en las fases de fotosíntesis CAM ante los cambios ambientales, al aumentar su fijación diurna por RUBIS$\mathrm{CO}$ cuando el agua es abundante, o disminuir el intercambio gaseoso de las fases nocturnas ante la sequía (Dodd et al., 2002; Andrade et al., 2009).

Con el uso cada vez más común de los isótopos estables, se sabe que la PEPc como primer aceptor de $\mathrm{CO}_{2}$, en plantas $\mathrm{C}_{4}$ y CAM, no discrimina entre el isótopo pesado $\left({ }^{13} \mathrm{C}\right)$ y el ligero del carbono $\left({ }^{12} \mathrm{C}\right)$ contenidos en el $\mathrm{CO}_{2}$ atmosférico $\left({ }^{13} \mathrm{CO}_{2}\right.$ y $\left.{ }^{12} \mathrm{CO}_{2}\right)$; a diferencia de RUBISCO que discrimina al isótopo pesado, por lo que las plantas CAM y $\mathrm{C}_{4}$ tienen una firma isotópica particular que las distingue de las $\mathrm{C}_{3}$ (Benzing, 2000). Dicha firma es el cociente de ${ }^{13} \mathrm{C}$ sobre ${ }^{12} \mathrm{C}$ de la muestra, con respecto al cociente en un estándar, y se conoce como delta ${ }^{13} \mathrm{C}$ (Santiago et al., 2005; Andrade et al., 2007).

\section{Efectos del ambiente sobre la fisiología de las bromeliá- ceas epífitas}

Cuando el microambiente de las epífitas cambia, los procesos fisiológicos de estas plantas son directamente afectados (Ruzana y Ainuddin, 2011). De manera que ante cualquier leve variación en el ambiente, tanto el proceso fotosintético como el crecimiento de las plantas cambian (Schurr et al., 2006). Lo anterior muestra que las epífitas tienen rangos de tolerancia estrechos a cambios en el ambiente; por lo que, la mayor diversidad y abundancia de especies epífitas se encuentran en los bosques húmedos, como los encinares y el mesófilo de montaña (Espejo-Serna et al., 2007), debido a la distribución uniforme de la lluvia durante el año (Benzing, 1998).

Una combinación de estrés por baja disponibilidad de agua y alta radiación solar puede causar fotoinhibición (reducción en la fotosíntesis debido al exceso de luz; DemmigAdams y Adams, 1992; Hamilton et al., 1995). Por ello, en las bromeliáceas epífitas han evolucionado una gran diversidad de estructuras de carácter adaptativo y mecanismos fisiológicos (formación de tanques para la captación de agua, suculencia foliar, tricomas foliares especializados en la absorción de agua y nutrimentos, así como la fotosíntesis CAM), que les han permitido adaptarse con éxito al ambiente epífito caracterizado por pulsos cortos de agua, altas intensidades lumínicas e inestabilidad de las ramas de los forofitos (árboles sobre los que crecen) ante los vientos fuertes (Benzing, 1990, 2000).

Entre las principales respuestas fisiológicas de las bromeliáceas epífitas a cambios en los factores ambientales, como la disponibilidad de agua, la radiación solar y la temperatura, se encuentran una reducción en: la tasa de asimilación de $\mathrm{CO}_{2}$, la magnitud de la acidificación nocturna (expresada como un delta de acidez), el contenido relativo de agua, el potencial hídrico y la eficiencia cuántica del fotosistema II; así como cambios en las concentraciones de pigmentos y en la señal isotópica de ${ }^{13} \mathrm{C}$, como resultado de la reducción del tiempo de apertura estomática y de la actividad enzimática. Todas estas respuestas se reflejan directamente en el crecimiento y desarrollo de estas plantas (Martin, 1994; Winter y Holtum, 2002; Andrade, 2003; Graham y Andrade, 2004; Cervantes et al., 2005; Cach-Pérez, 2008; González-Salvatierra et al., 2010; Reddy et al., 2010; Reyes-García et al., 2012).

Respuestas ante la baja disponibilidad de agua. Cuando hay un déficit hídrico para las plantas, la tasa de asimilación de $\mathrm{CO}_{2}$ se puede reducir considerablemente, ya que la conductancia estomática disminuye (Lambers et al., 1998; Stancato et al., 2001). En la figura 1 se muestra el efecto de la estacionalidad en la disponibilidad de agua sobre la variación diaria de la acidez tisular de cuatro especies de bromeliáceas epífitas, en tres tipos de vegetación diferentes (esta estacionalidad es utilizada como una medida indirecta de la fotosíntesis al relacionarse con la cantidad de $\mathrm{CO}_{2}$ fijado por las plantas durante la noche; Cach-Pérez, 2008; González-Salvatierra, 2009; Ricalde et al., 2010). En la temporada de lluvias, la acidez tisular es alta en las cuatro especies, comparada con la temporada de sequía. Durante 


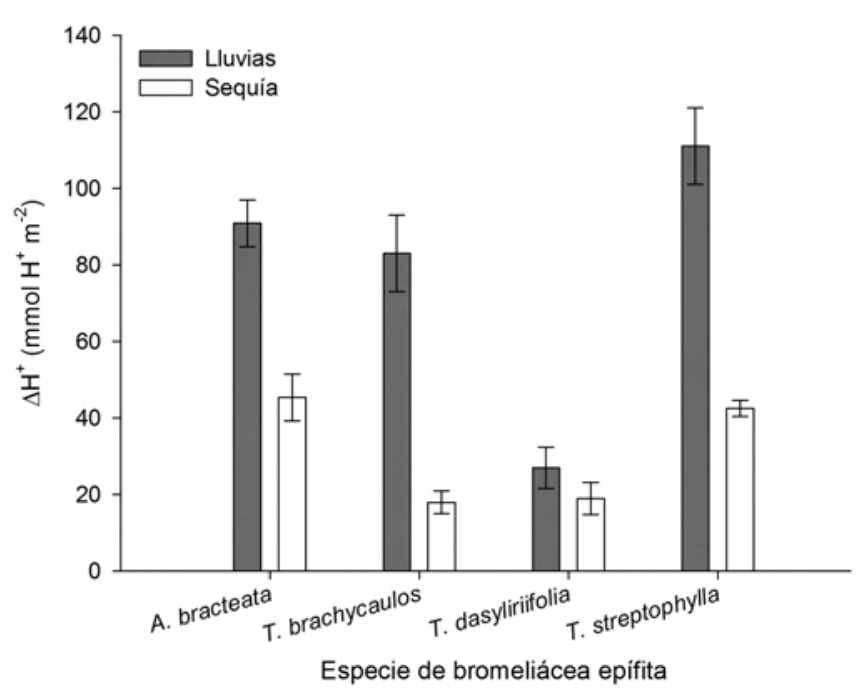

Figura 1. Acidez tisular (delta) durante la temporada de lluvias y sequía en cuatro especies de bromeliáceas epífitas en tres tipos de vegetación en la Península de Yucatán: Aechmea bracteata y Tillandsia brachycaulos en la selva baja caducifolia; T. dasyliriifolia en el matorral de duna costera y T. streptophylla en el manglar de Petén (Datos integrados de: Cach-Pérez, 2008; González-Salvatierra, 2009; Ricalde et al., 2010).

este periodo, la variación diurna de acidez se reduce de un $30 \%$ a un $78 \%$ (en Tillandsia dasyliriifolia y T. brachycaulos, respectivamente), lo que limita la producción de azúcares utilizados para el mantenimiento, crecimiento y desarrollo de las plantas.

El incremento en la precipitación total anual que reciben los diferentes hábitats en los que se encuentran las bromeliáceas epífitas favorece el aumento en la acidez tisular nocturna de estas plantas, tanto en sitios secos como en sitios húmedos (Figura 2); por lo que bajo escenarios de cambio climático, donde se proyectan reducciones en la cantidad de precipitación, la acidez tisular, y por tanto, la fijación de $\mathrm{CO}_{2}$, se verán afectadas negativamente. Griffiths et al. (1989) compararon la tasa de fijación de $\mathrm{CO}_{2}$, la acidez tisular, la tasa de transpiración y el potencial hídrico foliar en Tillandsia flexuosa durante las temporada de lluvia y sequía en el norte de Venezuela, y encontraron que los parámetros fotosintéticos fueron menores en el periodo de sequía comparado con el de lluvias, aún cuando el potencial hídrico varió únicamente de $-0.2 \mathrm{MPa}$ a $-0.3 \mathrm{MPa}$ entre el periodo de lluvias y sequía, respectivamente.

También, Lange y Medina (1979) mostraron que la fijación de $\mathrm{CO}_{2}$ en hojas de Tillandsia recurvata responde a variaciones en las condiciones de humedad del aire, pero el potencial hídrico foliar no muestra cambios. En esta especie, el aire seco provoca un incremento de la resistencia estomática a la difusión de $\mathrm{CO}_{2}$, mientras que con aire húmedo, la resistencia decrece; asimismo, existe un cierre estomático durante la noche en respuesta a la variación en el déficit de presión de vapor de agua del aire (DPV), por lo que la pérdida nocturna total de agua foliar fue menor a bajo DPV que a alto DPV (Lange y Medina, 1979). El efecto negativo del DPV también se ha documentado en T. stricta, ya que la asimilación diaria de $\mathrm{CO}_{2}$ se redujo de $33 \mathrm{mmol} \mathrm{m}^{-2} \mathrm{~s}^{-1}$ hasta $11 \mathrm{mmol} \mathrm{m}^{-2} \mathrm{~s}^{-1}$ con el incremento en el DPV, además de que la fase II de CAM no pudo ser observada (Reinert, 1995).

En las bromeliáceas epífitas con fotosíntesis $\mathrm{C}_{3}$, la falta de agua tiene efectos similares. Las hojas de Tillandsia heterophylla, bajo condiciones controladas, dejaron de fijar $\mathrm{CO}_{2}$ después de 14 días sin recibir agua. El contenido relativo de agua, el potencial osmótico y la eficiencia en el uso del agua también se redujeron de manera importante durante este periodo de sequía (Cach-Pérez, 2008). Por lo tanto, una reducción en la disponibilidad de agua en forma de lluvia, neblina o rocío, como lo predicen los modelos de cambio climático para las zonas tropicales (IPCC, 2007), podrían tener un efecto negativo importante sobre la asimilación de $\mathrm{CO}_{2}$ por parte de las bromeliáceas epífitas, y por consiguiente, sobre su crecimiento y supervivencia.

El efecto negativo de la baja disponibilidad de agua no es tan evidente en especies de bromeliáceas de hábitos terrestres, dada su menor sensibilidad a la variación ambiental. Por ejemplo, Bromelia karatas no presentó variación significativa en su potencial hídrico foliar a lo largo del año, aún con la reducción en la disponibilidad de agua durante la temporada de sequía en la Península de Yucatán, México; por lo contrario, una alta acidez tisular se mantiene durante esta época del año (González-Salvatierra, 2009; Ricalde et al., 2010 González-Salvatierra et al., 2013). Sin embargo, en otras especies, como $B$. humilis, las variaciones estacionales en la actividad de CAM (medida como la acumulación nocturna de ácidos orgánicos), se deben a variaciones en la precipitación en una región semiárida de Venezuela. En esta

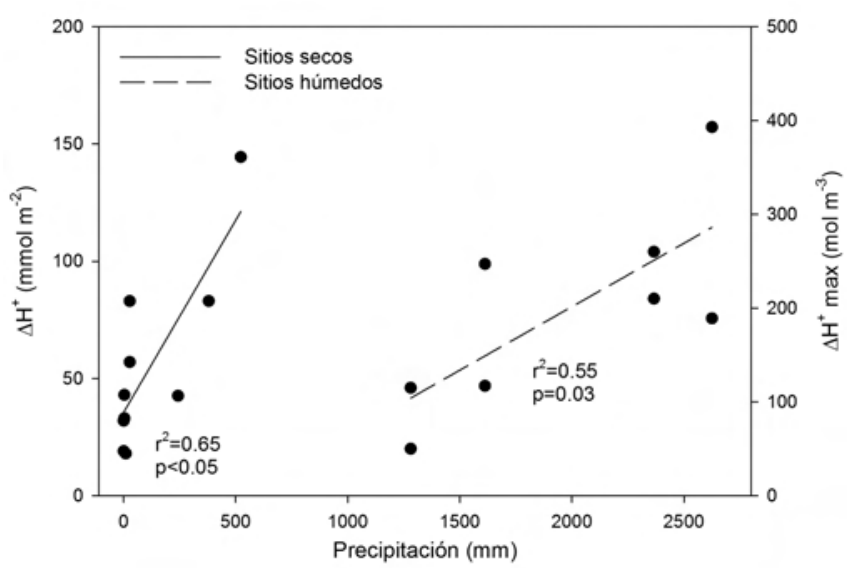

Figura 2. Variación en la acidez tisular (delta) promedio en bromeliáceas epífitas de bosques secos y acidez tisular máxima en bosques húmedos, en respuesta al incremento de la precipitación (Datos integrados de: Smith et al., 1986b; Cach-Pérez, 2008; González-Salvatierra, 2009; Ricalde et al., 2010). 
especie, la actividad de CAM fue alta durante la temporada de lluvia, se redujo al final de ésta, y fue baja durante la sequía (Medina et al., 1986).

Respuesta ante la variación en temperatura. Se podría esperar que un incremento leve de la temperatura ambiental durante la noche pudiera reflejar un aumento en la asimilación de $\mathrm{CO}_{2}$ en las epífitas CAM, debido a que la actividad de la PEPc se incrementaría. Esto se ha demostrado bajo condiciones controladas en las especies Tillandsia flexuosa y Aechmea nudicaulis en la isla de Trinidad (Smith et al., 1986b; Griffiths et al., 1989). Sin embargo, el incremento de la temperatura en unos pocos grados por arriba de la temperatura nocturna óptima para la fotosíntesis puede reducir el suministro de PEPc para la fijación nocturna de $\mathrm{CO}_{2}$ y favorecer la respiración, además de que puede tener un efecto desecante sobre el tejido foliar. Esto último se debe a que un incremento en la temperatura eleva el déficit de saturación de vapor de agua entre el aire que rodea a las hojas y el aire dentro de la cámara subestomática, lo que provoca una reducción de la conductancia estomática para evitar la pérdida de agua (Griffiths et al., 1986). En consecuencia, debido a la reducción en el suministro en PEPc y el cierre estomático, la cantidad de $\mathrm{CO}_{2}$ disponible para la síntesis de ácidos orgánicos disminuye.

Con el incremento de la temperatura ambiental en $1.5^{\circ} \mathrm{C}$, las hojas de la bromeliácea epífita Aechmea aquilega reducen la cantidad de $\mathrm{CO}_{2}$ fijado durante la noche en un $86 \%$, lo que se refleja en una disminución en la cantidad de ácidos orgánicos acumulados en el tejido vegetal de hasta un 54\% (Griffiths et al., 1986). A una temperatura nocturna de entre 15 y $17{ }^{\circ} \mathrm{C}$, las hojas de Tillandsia recurvata tienen tasas de asimilación de $\mathrm{CO}_{2}$ altas, mientras que a temperaturas mayores de $26{ }^{\circ} \mathrm{C}$, o cercanas a los $26{ }^{\circ} \mathrm{C}$, la asimilación de $\mathrm{CO}_{2}$ se inhibe; lo mismo se observó para la epifita T. usneoides, la cual tuvo una temperatura óptima de asimilación entre los 15 y $20{ }^{\circ} \mathrm{C}$, pero en temperaturas fuera de este rango hubo una reducción en la asimilación de carbono (Medina, 1987). Además, la baja acumulación nocturna de ácidos orgánicos causa una reducción en la fuente de hexosas para el crecimiento y reproducción de las bromeliáceas epífitas. Se ha observado que en respuesta a la variación en la temperatura ambiental, las plantas CAM muestran cierta termoplasticidad, o al menos, una aclimatación gradual a las temperaturas sub-óptimas iniciales (Martin, 1994).

En cuanto a las bromeliáceas epífitas con fotosíntesis $\mathrm{C}_{3}$, la asimilación de $\mathrm{CO}_{2}$ se reduce en Vriesea amazonica cuando la temperatura diurna excede $\operatorname{los} 35^{\circ} \mathrm{C}$ en la Isla de Trinidad (Griffiths et al., 1986). Algo similar es observado en Guzmania monostachia en el mismo lugar, ya que mostró una disminución en su tasa fotosintética cuando la temperatura ambiental superó $\operatorname{los} 31^{\circ} \mathrm{C}$ durante el día (Lüttge et al., 1986a). Estos datos muestran que la temperatura óptima para la fotosíntesis $\mathrm{C}_{3}$ en bromeliáceas epífitas es baja, si se consideran los ambientes tropicales en los que pueden ser encontradas; sin embargo, se ha observado un óptimo de temperatura que también es bajo para especies CAM como Tillandsia recurvata y T. utriculata en selvas tropicales, aunque dada la termoplasticidad ya mencionada, especies como T. usneoides pueden asimilar $\mathrm{CO}_{2}$ en un rango de temperatura mayor que especies $\mathrm{C}_{3}$ (Medina et al., 1977; Martin, 1994).

Estratificación vertical de la luz. Las epífitas pueden crecer bajo diferentes condiciones de luz, desde casi totalmente expuestas al sol en ramas superiores de sus hospederos, hasta la sombra profunda de la base del tallo de los mismos (Hietz y Briones, 2001). Por ello, Pittendrigh (1948) dividió a las bromeliáceas en tres grupos relacionados con sus necesidades de luz: plantas expuestas, de sol (medianamente expuestas) y tolerantes a la sombra. El autor propone que las plantas tolerantes a la sombra, en realidad no necesitan de la sombra, sino que requieren la alta humedad del sotobosque o estratos inferiores del dosel. De esta forma, se pueden encontrar especies creciendo tanto en sitios sombreados, como en sitios con una alta exposición a la luz, como es el caso de Guzmania monostachia que puede tolerar tanto la sombra hasta una exposición del $60 \%$ del total de radiación solar incidente (Maxwell et al., 1992).

En un experimento en el que se sometió a Tillandsia heterophylla a diferentes condiciones de luz (entre el 20\% y $90 \%$ del total de radiación solar incidente), Cach-Pérez (2008) encontró que plantas expuestas al 40\% del total de luz incidente, presentaron una mayor tasa de asimilación de $\mathrm{CO}_{2}$, altas tasas de transporte de electrones, y mayor capacidad de recuperación después de periodos prolongados de sequía, al compararse con plantas que crecieron al $20 \%$ y $90 \%$ del total de radiación solar incidente. Otra especie, T. usneoides, mostró alta acumulación nocturna de ácidos orgánicos creciendo con alta incidencia de flujo de fotones para la fotosíntesis bajo condiciones de laboratorio; sin embargo, datos de campo mostraron tasas de crecimiento similares bajo un amplio rango de intensidades lumínicas (Martin et al., 1985).

Los individuos expuestos a la radiación solar en lo alto del dosel presentan también un incremento en la temperatura foliar, que a su vez influye sobre el crecimiento vegetal debido a que el microclima alrededor de la planta se vuelve más caliente y seco (Ruzana y Ainuddin, 2011). En consecuencia, las hojas de las plantas presentan fotoinhibición cuando están sujetas a irradiaciones mayores (hasta en un $76 \%$ ) a las que usualmente reciben (Stancato et al., 2002). Generalmente, el aparato fotosintético se protege de la alta radiación solar reduciendo el contenido de clorofila total y manteniendo alta proporción de clorofila a:b (Björkman, 1981). Sin embargo, a pesar de que algunas especies de bromeliáceas toleran un amplio rango de radiación solar, y en consecuencia muestran una amplia variación en el conteni- 


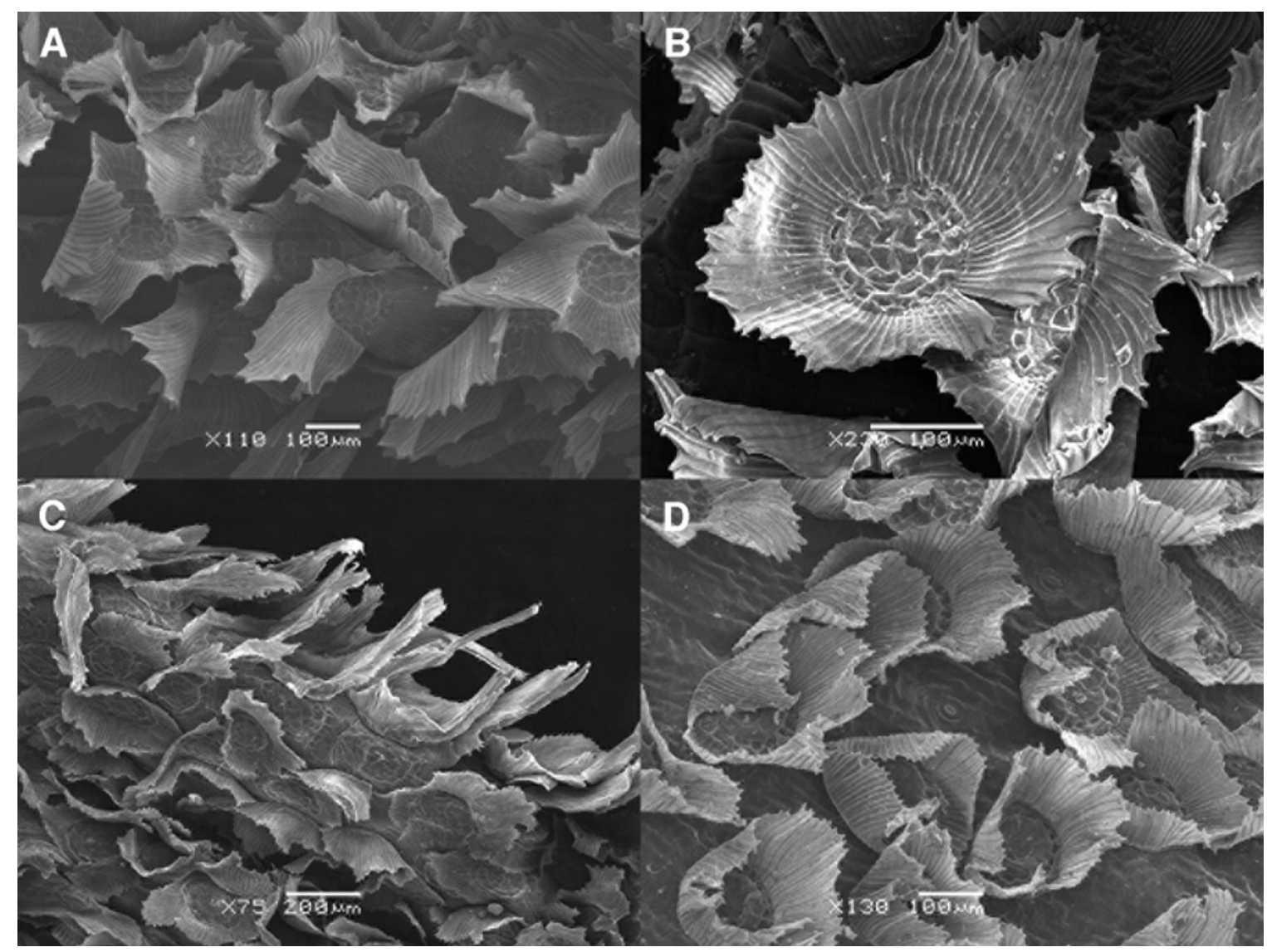

Figura 3. Imágenes al microscopio electrónico de barrido que muestran: A) vista general de los tricomas foliares de Tillandsia recurvata; B) acercamiento a los tricomas foliares de la misma especie; C) vista general de los tricomas foliares de T. schiedeana y D) los estomas debajo de las alas levantadas de los tricomas foliares en T. streptophylla.

do de clorofila, la proporción clorofila a:b parece no variar considerablemente (Martin, 1994).

Se ha observado un decremento en la eficiencia cuántica del fotosistema II con el incremento de la cantidad de luz incidente sobre las plantas (Graham y Andrade, 2004; Sarvikas et al., 2006; Cach-Pérez, 2008; González-Salvatierra, 2009). Por ello, en las bromeliáceas, al igual que en otras plantas, han evolucionado estrategias que les permiten afrontar el exceso de energía recibido en el aparato fotosintético, disipando la energía excedente en forma de calor (disipación no fotoquímica, NPQ por sus siglas en inglés), o re-emitirla como radiación electromagnética en un proceso conocido como fluorescencia de la clorofila (Maxwell y Johnson, 2000). Asimismo, aparte del aumento de la actividad antioxidante, en Tilllandsia brachycaulos se presenta una correlación positiva entre el contenido de antocianinas en las hojas y la exposición al sol (González-Salvatierra, 2010).

Estas estrategias parecen no ser necesarias en especies de bromeliáceas de hábitos terrestres. Por ejemplo, plantas de Ananas comosus tienen tasas de fijación nocturnas de $\mathrm{CO}_{2}$ altas cuando crecen bajo condiciones de alta radiación solar, comparadas con plantas que crecen en la sombra, con su tasa máxima de asimilación por arriba de los $1,500 \mu \mathrm{mol} \mathrm{m} \mathrm{m}^{-2} \mathrm{~s}^{-1}$ (Nose et al., 1977). De hecho, especies del género Ananas que crecen naturalmente en sitios sombreados tienen tasas fotosintéticas altas cuando crecen en alta irradiación (Keller y Lüttge, 2005). Las especies de bromeliáceas terrestres de sotobosque están sujetas a un régimen de irradiación cambiante y pueden usar las irradiaciones altas eficientemente cuando se presentan (Medina et al., 1994; Skillman y Winter, 1997). Esto sugiere que las bromeliáceas terrestres son menos sensibles a un rango de irradiación mayor que las bromeliáceas de hábito epífito (Martin, 1994; González-Salvatierra et al., 2013).

\section{Efectos del ambiente sobre la anatomía y morfología}

La influencia del microambiente sobre las plantas puede ser observada no sólo a nivel fisiológico, sino también a nivel morfológico y anatómico, ya sea reflejado en las diferentes orientaciones de las hojas de un mismo individuo o entre individuos de una misma especie bajo diferentes condiciones microambientales (Camacho y Bellefleur, 1996; Silva et al., 
1999). La morfología de las epífitas ha sufrido una adaptación para aprovechar los recursos disponibles en el ambiente donde crecen. Una de las modificaciones principales en las bromeliáceas epífitas son los tricomas foliares, especializados en la absorción de agua y nutrimentos (Benzing, 2000). La cubierta y densidad de los tricomas varían de acuerdo a la especie, pero en general son más abundantes en especies atmosféricas que en especies con tanque (Reyes-García et al., 2008b; Lüttge, 2010). Además, los tricomas se encuentran asociados a los estomas, a los que proveen protección contra la pérdida excesiva de agua, aunque también pueden limitar el intercambio de gases debido a que las células externas (alas) pueden cubrir completamente a los estomas (Figura 3), y favorecen la formación de una película de agua cuando la hoja está húmeda, lo que puede limitar el intercambio de gases (Benzing y Renfrow, 1971; Lüttge et al., 1986a; Martin, 1994). Sin embargo, una relación directa entre una mayor cubierta y tamaño de los tricomas, y una disminución en el intercambio de gases en especies de Tillandsia no ha sido encontrada (Benz y Martin, 2006).

Durante el desarrollo de las hojas, el microambiente puede afectar la densidad de estomas y tricomas de la hoja madura, ya que las hojas que crecen expuestas a la luz tendrán mayor número de estomas que las hojas que crecen bajo sombra (Reich, 1984). Pero la densidad de estas estructuras no sólo depende de la cantidad de radiación solar que recibe la planta, sino que también del área foliar, orientación de la hoja y la cantidad de agua que la planta recibe. De esta forma, cuando las condiciones de luz en las que crece una planta cambian (la intensidad luminosa se reduce o aumenta), se pueden encontrar variaciones en el número de estomas y tricomas entre hojas viejas (que la planta tenía antes del cambio) y hojas nuevas (producidas bajo las nuevas condiciones lumínicas; Figura 4A). Con esto se hace evidente la relación positiva entre el incremento de la cantidad de luz incidente y la densidad de estomas en especies (Figura 4B), como Tillandsia heterophylla (Cach-Pérez, 2008).

La importancia de la densidad de tricomas en las bromeliáceas radica en que, además del papel que juegan en la absorción de agua y nutrimentos, influyen en la regulación de la transpiración foliar y en la fotoprotección. En altas densidades, los tricomas actúan como un filtro difusivo que refleja parte de la luz incidente y disminuye la radiación solar que llega a la superficie de las hojas (Benzing y Renfrow, 1971; Lüttge et al., 1986b; Larcher, 2003). Sin embargo, en ocasiones, la cantidad de luz reflejada por los tricomas puede no ser la suficiente para la fotoprotección (Pierce et al., 2001). Por otra parte, el papel de los tricomas no se limita únicamente a la fotoprotección, ya que la densidad de éstos también puede influir sobre la densidad de los estomas, así como en el tamaño del poro estomático y el grosor de la capa límite. Por ejemplo, si la densidad estomática aumenta, el tamaño del poro estomático tenderá a disminuir y la capa límite será más estable (Nowak y Martin, 1997).

Por tanto, la composición (estructura y disposición) de los tricomas y de los estomas en las hojas de las bromeliáceas epifitas determina la forma en que capturan recursos y brinda a éstas ciertas ventajas sobre otras plantas (absorción de agua y nutrientes de la atmósfera, gran capacidad de control estomático en respuesta al ambiente). Estas características pueden ayudar a explicar por qué la bromeliáceas epifitas están presentes en un amplio rango de hábitats con microclimas altamente contrastantes (Benzing, 2000).

En las bromeliáceas epífitas, cambios en la anatomía y la fisiología foliar pueden interpretarse en el contexto de estrategias para "evitar la sequía" o para "tolerar la sequía" (Schmidt y Zotz, 2001). Esta idea se confirma al notar cómo epífitas más grandes aprovechan los periodos de lluvia gra-
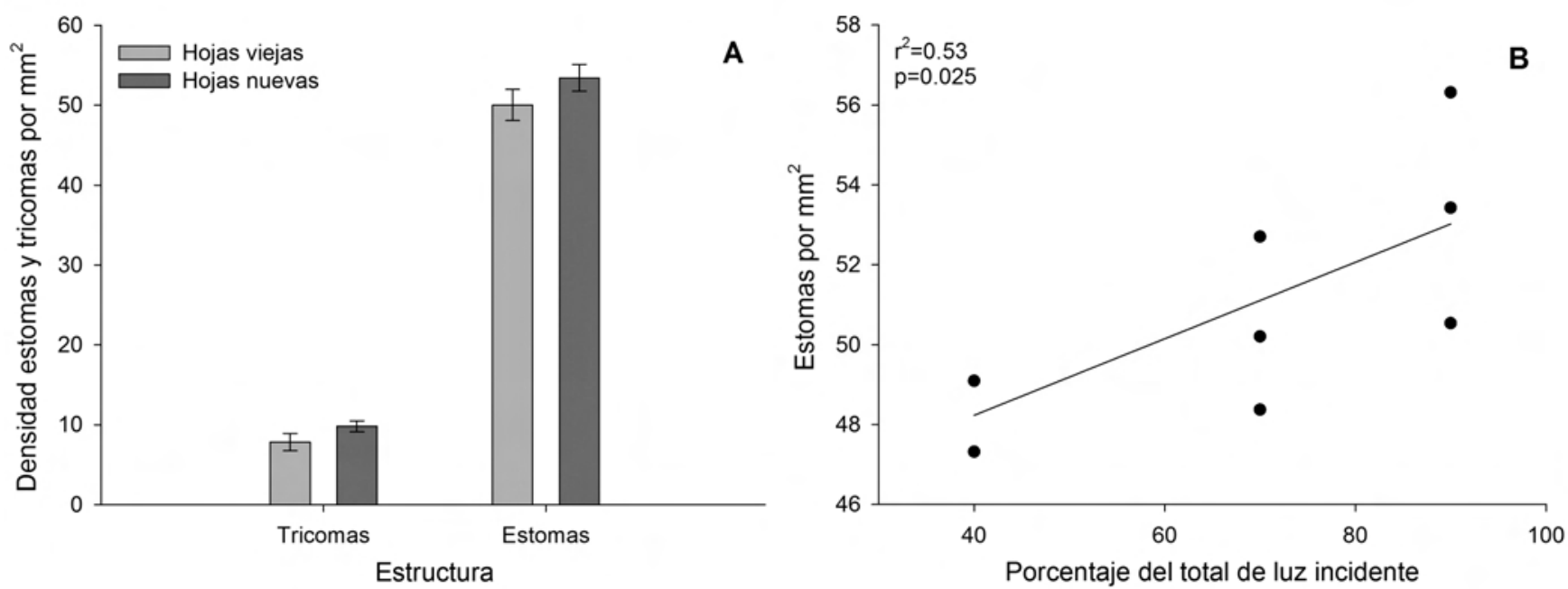

Figura 4. A) Variación en el número de estomas y tricomas bajo incremento de radiación solar incidente en hojas viejas (antes del incremento de la radiación) y nuevas (producidas después del incremento de la radiación) de Tillandsia heterophylla. B) Relación de la densidad de estomas con el incremento en la radiación solar incidente en hojas de T. heterophylla (Cach-Pérez, 2008). 
cias a un tanque eficiente en la captación y almacenamiento de agua, y evitan déficits hídricos de sus tejidos mediante un rápido cierre estomático después de que el agua del tanque se ha agotado (evitan la sequía). En general, las epífitas que recurren a esta estrategia han desarrollado una alta resistencia estomática y esclerofilia, lo que les permite conservar y/o almacenar agua para evitar la sequía. En contraste, la reducción en la conductancia estomática relacionada con la sequía de plantas pequeñas es relativamente menos pronunciada, lo que se interpreta como tolerancia a la sequía. Además, las epífitas tolerantes a la sequía pueden detener su crecimiento y evitar una severa desecación citoplasmática, hasta que puedan rehidratarse, momento en el que el crecimiento se reanuda (Zotz y Hietz, 2001; Reinert, 1998).

\section{Consecuencias del impacto del cambio climático sobre las bromeliáceas epífitas}

Las epífitas incrementan notablemente el inventario local de recursos de una comunidad (metabolitos secundarios, sitios de refugio, anidación y alimentación, entre otros). Por lo tanto, los cambios a nivel fisiológico, anatómico y morfológico que podrían sufrir las bromeliáceas epífitas (y las epífitas en general), ante el cambio en las condiciones de temperatura y humedad del aire relacionadas con el cambio climático, tendrían implicaciones importantes; primero sobre las plantas en sí mismas (poniendo en riesgo su supervivencia), y segundo, sobre los ecosistemas en los que habitan, al desaparecer parte importante de la biomasa de éstos, y por tanto, los nutrimentos que aportan al sistema, así como por su fuerte influencia sobre la fauna del dosel (Zotz, 2013).

La distribución de las plantas se rige por las condiciones climáticas favorables para ellas; por ejemplo, la temperatura influye sobre los rangos metabólicos (reducción en el suministro de PEPc), la luz condiciona la fotosíntesis, y ambas influyen sobre la apertura estomática que permite la transpiración, de acuerdo a déficits de vapor de agua atmosféricos y del grosor de la capa límite foliar (dada por el tamaño de la hoja y la velocidad del viento; Benzing, 1998; Lambers et al., 1998). Por lo tanto, si existen cambios en las condiciones climáticas en general, podría esperarse, como consecuencia, que las especies sigan una de tres alternativas: adaptarse, migrar o extinguirse, lo cual dependerá, en gran medida, de la rapidez con los que se presenten los cambios en el clima (Dawson et al., 2011).

Los tipos de vegetación que se reconocen en la actualidad se relacionan íntimamente con las condiciones climáticas en las que crecen. Villers y Trejo (2004) afirman que más de la mitad del territorio de la República Mexicana (entre 50\% y $57 \%$ ) cambiaría sus condiciones de temperatura y precipitación bajo escenarios de cambio climático, con tal magnitud, que el tipo de clima que existe hoy podría ser reclasificado de acuerdo a la clasificación de Köppen, modificada por García (García, 1973). Este cambio supone entonces, que las comunidades que se establecen actualmente en esas áreas serían afectadas y, por lo tanto, tendrían que cambiar su distribución de acuerdo a la variación climática. La mayoría de las proyecciones apuntan a que con un incremento en la temperatura y disminución en la precipitación, los bosques templados y húmedos serían los más afectados, lo que reduciría drásticamente su distribución (o incluso desaparecerían de algunas regiones), mientras que los desiertos y bosques secos podrían ampliar su distribución (Villers y Trejo, 2004).

En este sentido, el acoplamiento fino entre las epífitas y el clima, especialmente con la humedad, sugiere una alta probabilidad de que algunas especies sufran un fuerte impacto con el cambio en las condiciones ambientales en ciertas localidades (Reyes-García y Andrade, 2007; Reyes-García et al., 2008b), porque su capacidad para cambiar su distribución es menor que la de muchas especies de árboles. Entonces, las poblaciones de bromeliáceas que se distribuyen en la zonas bajas de los bosques de montaña podrían perder gran parte de sus individuos y especies si la disponibilidad de humedad disminuye (Gilmartin, 1973). Nadkarni y Solano (2002) demostraron experimentalmente, que la reducción en la disponibilidad de agua en bosques húmedos provoca que las epífitas (entre ellas las bromeliáceas) tengan una alta mortalidad de hojas, reducción en la producción de éstas, y en general, una reducción en la vida de las plantas. Además, gran parte de la estratificación vertical de las epífitas puede ser explicado por las diferencias en la sensibilidad de las semillas y plántulas en un gradiente vertical de humedad, resaltando la importancia de este factor sobre el establecimiento, distribución y supervivencia de las epífitas (Wagner et al., 2013).

Mondragón et al. (2004) encontraron que la población de Tillandsia brachycaulos en un selva baja de Yucatán, México, disminuyó después de un año seco (precipitación por debajo del promedio histórico anual), lo que demuestra que aún en esta especie adaptada a sitios relativamente secos, la reducción en la precipitación promedio ( $900 \mathrm{~mm}$ de media anual; Orellana et al., 2009), provoca cambios en la abundancia y posible distribución de especies. Hsu et al. (2012), mediante la simulación de variaciones en las condiciones ambientales producto del cambio climático en una región subtropical del este de Asia, demostraron que la distribución de las epífitas está altamente correlacionada con los distintos tipos de bosque en los que habitan, y proyectan que si la temperatura ambiental se incrementa entre 3.2 y $4.8^{\circ} \mathrm{C}$, el $78 \%$ de todas las especies epífitas perderían entre el $45 \%$ y el 58\% de su actual rango de distribución, y la altitud en la cual se distribuyen se incrementaría en promedio $400 \mathrm{~m}$, con respecto a su distribución actual.

Lo anterior es significativo si se considera que en un gradiente altitudinal el mayor número de especies epífitas se encuentra en la parte media del gradiente (Figura 5A), 

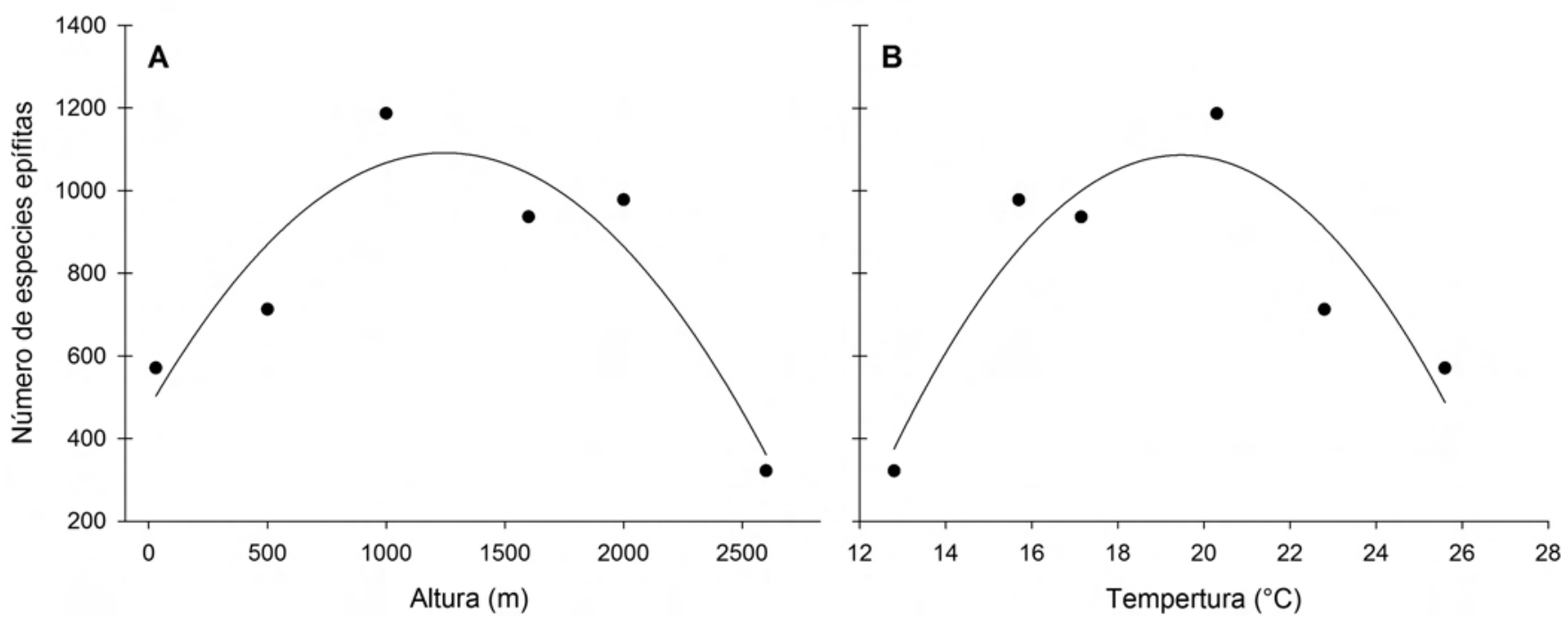

Figura 5. Distribución de epífitas en un gradiente altitudinal (A) y sus respectivas temperaturas ambientales (B) en Costa Rica (Cardelús et al., 2006).

donde las condiciones de temperatura y humedad del aire son óptimas para estas plantas (Figura 5B). Para especies tropicales que pudieran ser afectadas por un calentamiento del clima, los cambios en su distribución altitudinal parecen ser más factibles que los cambios latitudinales (Bush, 2002). Por tanto, las especies más afectadas por el cambio climático son las que presentan una distribución en zonas altas y húmedas, ya que no tendrán la posibilidad de migrar a regiones más elevadas. Las especies que tendrán la mayor posibilidad de soportar los cambios del clima son las especies que se distribuyen en las zonas bajas, fisiológicamente menos sensibles a variaciones ambientales y/o ampliamente distribuidas geográficamente (Colwell et al., 2008, Hsu et al., 2012).

Otro aspecto a considerar es que los cambios en la distribución, o incluso la desaparición local de especies, tendrían efectos negativos sobre el ciclo hidrológico y de nutrimentos en los ecosistemas. Las epífitas, y los suelos húmicos que generan y almacenan en el dosel, influyen en los procesos funcionales del bosque, dado que interceptan humedad y nutrimentos de la atmósfera, que de otro modo, podrían seguir diferentes rutas a través del sistema, o simplemente pasar a través de él (Benzing, 1998).

\section{Perspectivas}

Dada su alta sincronización con la atmósfera y su independencia del suelo, las epífitas son altamente vulnerables al cambio climático, en particular a la reducción en la disponibilidad de agua y al incremento en la temperatura, tanto en sitios relativamente secos, como en bosques húmedos de todo el continente americano. Sin embargo, esta vulnerabilidad puede ofrecer una buena oportunidad para estudiar el cambio climático a nivel regional. Epífitas como las bro- meliáceas pueden ser un buen modelo de estudio para identificar los posibles efectos del cambio climático por varias razones: su alta dependencia de la disponibilidad de agua en forma de lluvia, neblina o rocío (Andrade, 2003; Graham y Andrade, 2004; Mondragón et al., 2004; Reyes-García et al., 2008a), su tamaño pequeño y facilidad de manejo en campo y laboratorio, así como su rápida respuesta fisiológica ante variaciones ambientales.

Más estudios fisiológicos y ecológicos son necesarios para la modelación de los cambios en la distribución o desaparición de las bromeliáceas epífitas, así como para evaluar el efecto que tendría el cambio climático en la dinámica de los ecosistemas (ciclo de nutrimentos, producción de biomasa, captura de carbono, entre otros), y sus posibles efectos sobre grupos de especies estrechamente relacionados, tales como artrópodos, anfibios y reptiles. Asimismo, la investigación sobre la fisiología y ecología de epífitas también permitiría evaluar los servicios ambientales que los ecosistemas ofrecen y generaría los conocimientos básicos necesarios para estrategias de conservación, restauración y educación ambiental; lo cual conforma un conocimiento fundamental y urgente para la creación de una mayor conciencia sobre el cambio climático y el papel de las bromeliáceas epífitas en los ecosistemas.

\section{Agradecimientos}

A Eric Graham, Fernanda Ricalde, Claudia González-Salvatierra, Luis Simá y Roberth Us-Santamaría por su participación en nuestro laboratorio en los estudios sobre la fisiología y ecología de bromeliáceas epífitas. A Ivón Ramírez, Roger Orellana, Lucía Sanaphre, Edilia de la Rosa, Diana Cisneros, Saúl Hernández-Robinson, María Cabañas, Eduardo Chávez, Nahlleli Chilpa, Fernando Arellano y Mario León 
por comentarios a una versión previa del manuscrito. Al Dr. Ernesto Medina y un revisor anónimo por sus atinados comentarios y sugerencias. Al CONACyT por la beca de doctorado otorgada a M. J. Cach-Pérez (204451). Este estudio tuvo financiamiento parcial de los fondos SEP-CONACyT (proyecto: 80181), SEMARNAT (107916) y UC-MEXUS (2008-CL08-01).

\section{Literatura citada}

Andrade J.L. 2003. Dew deposition on epiphytic bromeliad leaves: an important event in a Mexican tropical dry deciduous forest. Journal of Tropical Ecology 19:479-488.

Andrade J.L. y Nobel P.S. 1996. Habitat, $\mathrm{CO}_{2}$ uptake and growth for the CAM epiphytic cactus Epiphyllum phyllanthus in a $\mathrm{Pa}-$ namanian tropical forest. Journal of Tropical Ecology 12:291306.

Andrade J.L. y Nobel P.S. 1997. Microhabitats and water relations of epiphytic cacti and ferns in a lowland Neotropical forest. Biotropica 29:261-270.

Andrade J.L., Cervera J.C. y Graham E.A. 2009. Microenvironments, water relations and productivity of CAM plants. En: De la Barrera E. y Smith W.K. Eds. Perspectives in Biophysical Plant Ecophysiology: A Tribute to Park S. Nobel, pp. 95-120, Universidad Nacional Autónoma de México, Centro de Enseñanza para Extranjeros, Centro de Investigaciones en Ecosistemas, Campus Morelia, México, D.F.

Andrade J.L., De la Barrera E., Reyes-García C., Ricalde M.F., Vargas-Soto G. y Cervera J.C. 2007. El metabolismo ácido de las crasuláceas: diversidad, fisiología ambiental y productividad. Boletín de la Sociedad Botánica de México 81:37-50.

Benz B.W. y Martin C.E. 2006. Foliar trichomes, boundary layers, and gas exchange in 12 species of epiphytic Tillandsia (Bromeliaceae). Journal of Plant Physiology 163:648-656.

Benzing D.H. y Renfrow A. 1971. The significance of photosynthetic efficiency to habitat preference and phylogeny among tillandsioid bromeliads. Botanical Gazette 132:19-30.

Benzing D.H. 1989. The mineral nutrition of epiphytes. En: Lüttge U. Ed. Vascular Plants as Epiphytes. Evolution and Ecophysiology, pp.167-199, Springer-Verlag, Berlín.

Benzing D. H. 1990. Vascular Epiphytes. General Biology and Related Biota. Cambridge University Press. Nueva York.

Benzing D.H. 1998. Vulnerabilities of tropical forest to climate change: The significance of resident epiphytes. Climate Change 39:519-540.

Benzing D.H. 2000. Bromeliaceae: Profile of an Adaptative Radiation. Cambridge University Press. Cambridge.

Björkman O.1981. Responses to different quantum flux densities. En: Lange O.L., Nobel P.S., Osmond C.B. y Ziegler H. Eds. Encyclopedia of Plant Physiology. New series 12A Physiological Plant Ecology, 57-107, Springer-Verlag, Berlín.

Bush M.B. 2002. Distributional change and conservation on the Andean flank: a palaeoecological perspective. Global Ecology and Biogeography 11:463-473.

Cach-Pérez M.J. 2008. Influencia ambiental sobre la fisiología y anatomía foliar de Tillandsia heterophylla, bromelia endémica de México. Tesis de Maestría en Ciencias. División de Posgrado, Instituto de Ecología, A. C. Xalapa. 80 pp.

Camacho M. y Bellefleur P. 1996. Aclimatación morfológica a la luz en seis especies arbóreas de los bosques montanos de Costa Rica. Revista de Biología Tropical 44:71-79.

Cardelús C.L., Colwell R.K. y Watkins J.E.Jr. 2006. Vascular epiphyte distribution patterns: explaining the mid-elevation richness peak. Journal of Ecology 94:144-156.

Cervantes S.E., Graham E.A. y Andrade J.L. 2005. Light microhabitats, growth and photosynthesis of an epiphytic bromeliad in a tropical dry forest. Plant Ecology 179:107-118.

Colwell R.K, Brehm G., Cardelús C.L., Gilman A.C. y Longino J.T. 2008. Global warming, elevational range of shifts, and lowland biotic attrition in the wet tropics. Science 322:258-261.

Dawson T.P., Jackson S.T., House J.I., Prentice I.C. y Mace G.M. 2011. Beyond predictions: biodiversity conservation in a changing climate. Science 332:53-58.

Demmig-Adams B. y Adams W.W.III 1992. Photoprotection and other responses to high light stress. Annual Review of Plant Physiology and Plant Molecular Biology 43:599-626.

Dodd A.N., Borland A.M., Haslam R.P., Griffiths H. y Maxwell K. 2002. Crassulacean acid metabolism: plastic, fantastic. Journal of Experimental Botany 53:569-580.

Espejo-Serna A., López-Ferrari A.R., Martínez-Correa N. y Pulido-Esparza V.A. 2007. Bromeliad flora of Oaxaca, Mexico: richness and distribution. Acta Botanica Mexicana 81:71-147.

García E. 1973. Modificaciones al Sistema de Clasificación Climática de Köppen: Para Adaptarlo a las Condiciones de la República Mexicana. Universidad Nacional Autónoma de México. México, D.F.

Gentry A.W. y Dodson C.H. 1987. Diversity and biogeography of neotropical vascular epiphytes. Annals of the Missouri Botanical Garden 74:205-233.

Gilmartin A.J. 1973. Transandean distributions of bromeliaceae in Ecuador. Ecology 54:1389-1393.

González-Salvatierra C. 2009. Antioxidantes y fotoprotección en dos especies con metabolismo ácido de las crasuláceas en una selva baja de Yucatán. Tesis de doctorado. Centro de Investigación Científica de Yucatán. Mérida, Yucatán. 177 pp.

González-Salvatierra C., Andrade J.L., Escalante-Erosa F., GarcíaSosa K. y Peña-Rodríguez L.M. 2010. Antioxidant content in two CAM bromeliad species as a response to seasonal light changes in a tropical dry deciduous forest. Journal of Plant Physiology 167:792-799.

González-Salvatierra C., Andrade, J.L., Orellana R., Peña-Rodríguez L.M. y Reyes-García C. 2013. Microambiente lumínico y morfología y fisiología foliar de Bromelia karatas (Bromeliaceae) en una selva baja caducifolia de Yucatán, México. Botanical Sciences 91: 75-84.

Graham E.A. y Andrade J.L. 2004. Drought tolerance associated with vertical stratification of two co-occurring epiphytic bromeliads in a tropical dry forest. American Journal of Botany 91:699-706.

Griffiths H., Smith J.A.C., Lüttge U., Popp M., Cram W.J., Diaz M., Lee H.S.J., Medina E., Schäfer C. y Stimmel K.H. 1989. Ecophysiology of xerophytic and halophytic vegetation of a coastal alluvial plain in northern Venezuela. IV. Tillandsia flexuosa Sw. and Schomburgkia humboltiana Reichb., epiphytic CAM plants. New Phytologist 111:273-282.

Griffiths H., Lüttge U., Stimmel K.H., Crook C.E., Griffiths N.M. y Smith J.A.C. 1986. Comparative ecophysiology of CAM and $\mathrm{C}_{3}$ bromeliad. III. Environmental influences on $\mathrm{CO}_{2}$ assimilation and transpiration. Plant, Cell and Environment 9:385-393. 
Hamilton L.S., Juvik J.O. y Scatena F. 1995. Tropical Mountain Cloud Forest. Springer-Verlag. Nueva York.

Helliker B.R. y Griffiths H. 2007. Toward a plant-based proxy for the isotope ratio of atmospheric water vapor. Global Change Biology 13:723-733.

Hietz P. y Briones O. 2001. Photosynthesis, chlorophyll fluorescence and within-canopy distribution of epiphytic ferns in a Mexican cloud forest. Plant Biology 3:279-287.

Hsu R.C.C., Tamis W.L.M., Raes N., de Snoo G.R., Wolf J.H.D., Oostermeijer G. y Lin S.H. 2012. Simulating climate change impacts on forest and associated vascular epiphytes in a subtropical island of East Asia. Diversity and Distributions 18:334-347.

IPCC. Intergovernmental Panel of Climate Change. 2007. Cambio climático 2007: Informe de síntesis. Contribución de los Grupos de trabajo I, II y III al Cuarto Informe de evaluación del Grupo Intergubernamental de Expertos sobre el Cambio Climático. IPCC, Ginebra.

Keller P. y Lüttge, U. 2005. Photosynthetic light-use by three bromeliads originating from shaded sites (Ananas ananassoides, Ananas comosus cv. Panare) and exposed sites (Pitcairnia pruinosa) in the medium Orinoco basin, Venezuela. Biologia Plantarum 49:73-79.

Kress W.J. 1986. The systematic distribution of vascular epiphytes: an update. Selbyana 9:2-22.

Lange O.L. y Medina E. 1979. Stomata of the CAM plant Tillandsia recurvata respond directly to humidity. Oecologia 40:357363.

Lambers H., Chapin F.S.III y Pons T.L. 1998. Plant Physiological Ecology. Springer-Verlag. Nueva York.

Larcher W. 2003. Physiological Plant Ecology: Ecophysiology and Stress Physiology of Functional Groups. $4^{\mathrm{a}}$ ed. Springer. Nueva York.

Lesica P. y Antibus R.K. 1990. The occurrence of mycorrhizae in vascular epiphytes of two Costa Rican rain forests. Biotropica 22:250-258.

Lugo A.E. y Scatena F.N. 1992. Epiphytes and climate change research in the Caribbean: a proposal. Selbyana 13:123-130.

Lüttge U., Stimmel K.H., Smith J.A.C. y Griffiths H. 1986a. Comparative ecophysiology of CAM and $\mathrm{C}_{3}$ bromeliads. II. Field measurements of gas exchange of CAM bromeliads in the humid tropics. Plant, Cell and Environment 9:377-383.

Lüttge U., Klauke B., Griffiths H., Smith J.A.C. y Stimmel K.H. 1986b. Comparative ecophysiology of CAM and $\mathrm{C}_{3}$ bromeliads. V. Gas exchange and leaf structure of the $\mathrm{C}_{3}$ bromeliad Pitcairnia integrifolia. Plant, Cell and Environment 9:411-419.

Lüttge U. 1989. Vascular Plants as Epiphytes. Evolution and Ecophysiology. Springer-Verlag, Berlín.

Lüttge U. 2010. Ability of crassulacean acid metabolism plants to overcoming interacting stresses on tropical environments. $A o B$ Plants. doi: 10.1093/aobpla/plq005.

Martin C.E. 1994. Physiological ecology of the Bromeliaceae. The Botanical Review 60:1-82.

Martin C.E., McLeod K.W., Eades C.A., y Pitzer A.F. 1985. Morphological and physiological responses to irradiance in the CAM epiphyte Tillandsia usneoides L. (Bromeliaceae). Botanical Gazette 146:489-494.

Maxwell K. y Johnson G.N. 2000. Chlorophyll fluorescence - a practical guide. Journal of Experimental Botany 51:659-668.

Maxwell C., Griffiths H., Borland A.M., Broadmeadow M.S.J. y McDavid C.R. 1992. Photoinhibitory responses of the epiphy- tic bromeliad Guzmania monostachia during the dry season in Trinidad maintain photochemical integrity under adverse conditions. Plant, Cell and Environment 15:37-47.

Medina E. 1987. Aspectos ecofisiológicos de plantas CAM en los trópicos. Revista de Biología Tropical 35:55-70.

Medina E., Delgado M., Troughton J. y Medina J.D. 1977. Physiological ecology of $\mathrm{CO}_{2}$ fixation in Bromeliaceae. Flora 166:137-152.

Medina E., Olivares E. y Diaz M. 1986. Water stress and light intensity effects on growth and nocturnal acid accumulation in a terrestrial CAM bromeliad (Bromelia humilis Jacq.) under natural conditions. Oecologia 70:441-446.

Medina E., Ziegler H., Lüttge U., Trimborn P. y Francisco M. 1994. Light conditions during growth as revealed by $\delta^{13} \mathrm{C}$ values of leaves of primitive cultivars of Ananas comosus, an obligate CAM species. Functional Ecology 8:298-305.

Mondragón D., Durán R., Ramírez I. y Valverde T. 2004. Temporal variation in the demography of the clonal epiphyte Tillandsia brachycaulos (Bromeliaceae) in the Yucatan Peninsula, Mexico. Journal of Tropical Ecology 20:189-200.

Nadkarni N.M. 1984. Epiphyte biomass and nutrient capital of a Neotropical elfin forest. Biotropica 16:249-256.

Nadkarni N.M. 1992. The conservation of epiphytes and their habitats: summary of a discussion at the international symposium on the biology and conservation of epiphytes. Selbyana 13:140142.

Nadkarni N. y Solano R. 2002. Potential effects of climate change on canopy communities in a tropical cloud forest: an experimental approach. Oecologia 131:580-586.

Nieder J., Prosperí J. y Michaloud G. 2001. Epiphytes and their contribution to canopy diversity. Plant Ecology 153:51-63.

Nose A., Shiroma M., Miyazato K. y Murayama S. 1977. Studies on matter production in pineapple plants: I. Effects of light intensity in light period on the $\mathrm{CO}_{2}$ exchange and $\mathrm{CO}_{2}$ balance of pineapple plants. Japanese Journal of Crop Science 46:580587.

Nowak E.J. y Martin C.E. 1997. Physiological and anatomical responses to water deficits in the CAM epiphyte Tillandsia ionantha (Bromeliaceae). International Journal Plant Sciences 158:818-826.

Orellana R., Espadas C., Conde C. y Gay C. 2009. Atlas. Escenarios de cambio climático en la Península de Yucatán. Centro de Investigación Científica de Yucatán, A.C., Universidad Nacional Autónoma de México, Consejo Nacional de Ciencia y Tecnología, Gobierno de Yucatán. Mérida.

Osmond C.B. 1987. Photosynthesis and carbon economy of plants. New Phytologist 106(suppl):161-175.

Pierce S., Maxwell K., Griffiths H. y Winter K. 2001. Hydrophobic trichome layers and epicuticular wax powders in Bromeliaceae. American Journal of Botany 88:1371-1389.

Pittendrigh C.S. 1948. The bromeliad-anopheles-malaria complex in Trinidad. I-The bromeliad flora. Evolution 2:58-89.

Reddy A.R., Rasineni G.K. y Raghavendra A.S. 2010. The impact of global elevated $\mathrm{CO}_{2}$ concentration on photosynthesis and plant productivity. Current Science 99:46-57.

Reich P.B. 1984. Leaf stomatal density and diffusive conductance in three amphistomatous hybrid poplar cultivars. New Phytologist 98:231-239.

Reinert F. 1995. On the Bromeliaceae of the restinga of Barra de Maricá in Brazil: environmental influences on the express ion 
of crassulacean acid metabolism. Ph.D. thesis, University of Newcastle. Newcastle. 234 pp.

Reinert F. 1998. Epiphytes: photosynthesis, water balance and nutrients. En Scarano F.R. y Franco A.C. Eds. Ecophysiological Strategies of Xerophytic and Amphibious Plants in the Neotropics. Series Oecologia Brasiliensis, Vol. IV. 87-108, Computer \& Publish Editoração Ltda., Río de Janeiro.

Reyes-García C. y Andrade J.L. 2007. Los isótopos estables del hidrógeno y el oxígeno en los estudios ecofisiológicos de plantas. Boletín de la Sociedad Botánica de México 80:19-28.

Reyes-García C., Mejia-Chang M. y Griffiths H. 2012. High but not dry: diverse epiphytic bromeliad adaptations to exposure within a seasonally dry tropical forest community. New Phytologist 193:745-754.

Reyes-García C., Griffiths H., Rincón E. y Huante P. 2008a. Niche differentiation in tank and atmospheric bromeliads of seasonally dry forest. Biotropica 40:168-175.

Reyes-García C., Mejía-Chang M., Jones G.D. y Griffiths H. 2008b. Water vapor isotopic exchange by epiphytic bromeliads in tropical dry forest reflects niche differentiation and climatic signals. Plant, Cell and Environment 31:828-841.

Ricalde M.F., Andrade J.L., Durán R., Dupuy J.M., Simá J.L., UsSantamaría R. y Santiago L.S. 2010. Environmental regulation of carbon isotope composition and crassulacean acid metabolism in three plant communities along a water availability gradient. Oecologia 164:871-880.

Ruzana A.M.S. y Ainuddin A.N. 2011. Epiphytic plant responses to light and water stress. Asian Journal of Plant Sciences 10:97107.

Santiago L.S., Silvera K., Andrade J.L. y Dawson T.E. 2005. El uso de isótopos estables en biología tropical. Interciencia 30:28-35.

Sarvikas P., Hakala M., Pätsikkä E., Tyystjärvi T. y Tyystjärvi E. 2006. Action spectrum of photoinhibition in leaves of wild type and $n p q 1-2$ and $n p q 4-1$ mutants of Arabidopsis thaliana. Plant and Cell Physiology 47:391-400.

Schurr U., Walter A. y Rascher U. 2006. Functional dynamics of plant growth and photosynthesis -from steady-state to dynamics- from homogeneity to heterogeneity. Plant, Cell and Environment 29:340-352.

Schmidt G. y Zotz G. 2001. Ecophysiological consequences on differences in plant size: in situ carbon gain and water relations of the epiphytic bromeliad, Vriesea sanguinolenta. Plant, Cell and Environment 24:101-111.

Silva H., Martínez J.P., Baginsky C. y Pinto M. 1999. Efecto del déficit hídrico en la anatomía foliar de seis cultivares de poroto. Revista Chilena de Historia Natural 72:219-235.
Skillman J.B. y Winter K. 1997. High photosynthetic capacity in a shade-tolerant crassulacean acid metabolism plant: Implications for sunfleck use, nonphotochemical energy dissipation, and susceptibility to photoinhibition. Plant Physiology 113:441-450.

Smith J.A.C., Griffiths H. y Lüttge U. 1986a. Comparative ecophysiology of CAM and $\mathrm{C}_{3}$ bromeliads. I. The ecology of the bromeliaceae in Trinidad. Plant, Cell and Environment 9:359376.

Smith J.A.C., Griffiths H., Lüttge U., Crook C.E., Griffiths N.M. y Stimmel K.H. 1986b. Comparative ecophysiology of CAM and $\mathrm{C}_{3}$ bromeliad. IV. Plant water relations. Plant, Cell and Environment 9:395-410.

Stancato G.C., Mazzafera P. y Buckeridge M.S. 2001. Effect of drought period on the mobilisation of non-structural carbohydrates, photosynthetic efficiency and water status in an epiphytic orchid. Plant Physiology and Biochemistry 39:1009-1016.

Stancato G.C., Mazzafera P. y Buckeridge M.S. 2002. Effects of light stress on the growth of the epiphytic orchid Cattleya forbesii Lindl. X Laelia tenebrosa Rolfe. Revista Brasileira de Botánica 25:229-235.

Villers L. y Trejo I. 2004. Evaluación de la vulnerabilidad en los ecosistemas forestales. En: Martínez J. y Fernández B.A. Comp. Cambio climático: Una Visión desde México, pp. 239254, Secretaría de Medio Ambiente y Recursos Naturales, Instituto Nacional de Ecología, México, D.F.

Wagner K., Bogusch W. y Zotz G. 2013. The role of the regeneration niche for the vertical stratification of vascular epiphytes. Journal of Tropical Ecology 29:277-290.

Winter K. y Holtum J.A.M. 2002. How closely do the $\delta^{13} \mathrm{C}$ values of crassulacean acid metabolism plants reflect the proportion of $\mathrm{CO}_{2}$ fixed during day and night? Plant Physiology 129:18431851 .

Winter K. y Smith J.A.C. 1996. Crassulacean Acid Metabolism: Biochemistry, Ecophysiology and Evolution. Springer, Berlín.

Zotz G. 2013. The systematic distribution of vascular epiphytes - a critical update. Botanical Journal of the Linnean Society 171:453-481.

Zotz G. y Asshoff R. 2009. Growth in epiphytic bromeliads: response to the relative supply of phosphorus and nitrogen. Plant Biology 12:108-113.

Zotz G. y Bader M.Y. 2009. Epiphytic plants in a changing worldglobal: Change effects on vascular and non-vascular epiphytes. Progress in Botany 70:147-170.

Zotz G. y Hietz P. 2001. The physiological ecology of vascular epiphytes: current knowledge, open questions. Journal of Experimental Botany 52:2067-2078. 